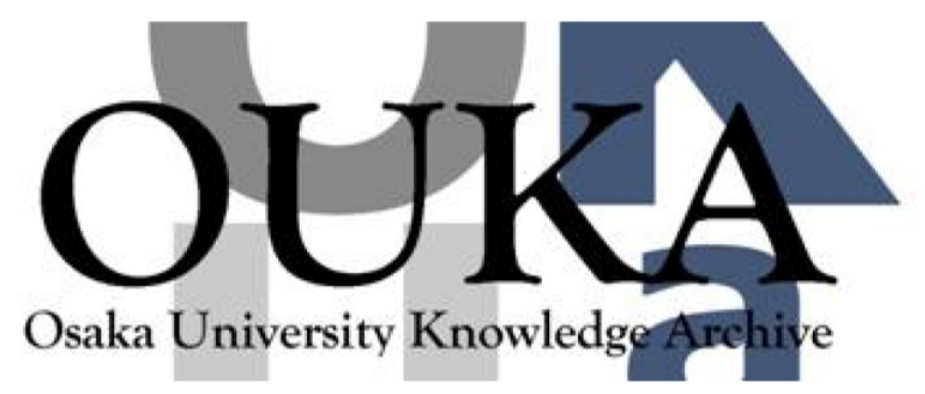

\begin{tabular}{|c|l|}
\hline Title & $\begin{array}{l}\text { Meaning of the field dependence of the } \\
\text { renormalization scale in Higgs inflation }\end{array}$ \\
\hline Author(s) & $\begin{array}{l}\text { Hamada, Yuta; Kawai, Hikaru; Nakanishi, Yukari } \\
\text { et al. }\end{array}$ \\
\hline Citation & Physical Review D. 95(10) p. 103524 \\
\hline Issue Date & $2017-05-31$ \\
\hline oaire:version & VoR \\
\hline URL & https://hdl. handle. net/11094/78739 \\
\hline rights & 02017 American Physical Society \\
\hline Note & \\
\hline
\end{tabular}

Osaka University Knowledge Archive : OUKA

https://ir. Library. osaka-u. ac. jp/

0saka University 


\title{
Meaning of the field dependence of the renormalization scale in Higgs inflation
}

\author{
Yuta Hamada, ${ }^{1,2, *}$ Hikaru Kawai, ${ }^{3, \dagger}$ Yukari Nakanishi, ${ }^{4, \$}$ and Kin-ya Oda ${ }^{4, \S}$ \\ ${ }^{1}$ KEK Theory Center, IPNS, KEK, Tsukuba, Ibaraki 305-0801, Japan \\ ${ }^{2}$ Department of Physics, University of Wisconsin, Madison, Wisconsin 53706, USA \\ ${ }^{3}$ Department of Physics, Kyoto University, Kyoto 606-8502, Japan \\ ${ }^{4}$ Department of Physics, Osaka University, Osaka 560-0043, Japan
}

(Received 26 October 2016; published 31 May 2017)

\begin{abstract}
We consider the prescription dependence of the Higgs effective potential under the presence of general nonminimal couplings. We evaluate the fermion loop correction to the effective action in a simplified Higgs-Yukawa model whose path integral measure takes simple form either in the Jordan or Einstein frame. The resultant effective action becomes identical in both cases when we properly take into account the quartically divergent term coming from the change of measure. Working in the counterterm formalism, we clarify that the difference between the prescriptions I and II comes from the counter term to cancel the logarithmic divergence. This difference can be absorbed into the choice of tree-level potential from the infinitely many possibilities, including all the higher-dimensional terms. We also present another mechanism to obtain a flat potential by freezing the running of the effective quartic coupling for large field values, using the nonminimal coupling in the gauge kinetic function.
\end{abstract}

DOI: 10.1103/PhysRevD.95.103524

\section{INTRODUCTION}

The Higgs inflation $[1,2]$ is one of the closest to the best fit point in the tensor-to-scalar ratio vs spectral-index plane among various inflation models [3]. The model requires rather a large nonminimal coupling $\xi \sim 10^{5-6}$ between the Higgs-squared $H^{\dagger} H$ and the Ricci scalar $\mathcal{R}$. ${ }^{1}$ On the other hand, the observed value of the Higgs mass $m_{H}=$ $125.09 \pm 0.24 \mathrm{GeV}[10]$ indicates that the standard model (SM) is at the criticality, that is, the Higgs potential becomes small and nearly flat when the Higgs-field value is close to the Planck-scale; see e.g. Refs. [11,12].

In the flat spacetime, the renormalized Higgs potential $V$ can be computed as a sum of the tree-level potential $V_{\mathrm{R}}$ and the loop correction $\Delta V_{\mathrm{R}}$, both of them being finite but depending on the renormalization scale $\mu$, in the counterterm formalism. Since $V$ is independent of $\mu$, we may choose $\mu$ arbitrarily. A convenient choice is $\mu \sim \varphi$, where $\varphi$ is the Higgs field value. This choice minimizes $\Delta V_{\mathrm{R}}$, and then $V$ can be approximated by the tree-level potential: $\left.V \simeq V_{\mathrm{R}}\right|_{\mu=\varphi}$.

\footnotetext{
yhamada@wisc.edu

†hkawai@gauge.scphys.kyoto-u.ac.jp

nakanishi@het.phys.sci.osaka-u.ac.jp

\$odakin@phys.sci.osaka-u.ac.jp

${ }^{1}$ The earlier model [1,4-6], without the Einstein-Hilbert action at the tree level, requires $\xi \sim 10^{34}$ [2]. In Ref. [1], the authors have also studied the Higgs inflation model in Ref. [2] with essentially the same parameter $\xi \sim 10^{4}$ and $\lambda \sim\left(\xi / 10^{5}\right)^{2} \sim 10^{-2}$. See also Refs. [7,8] for inflation with the nonminimal coupling, and Ref. [9] for a possible issue with the large nonminimal coupling.
}

When we couple this system with gravity, in general, there arise corrections from the nonrenormalizable couplings such as $\xi$. Under the presence of $\xi$, it has been said that there are two different "prescriptions" in which the renormalized Higgs potential is approximated by the treelevel one with [13-15]

$$
\mu \sim \begin{cases}\frac{\varphi}{\sqrt{1+\xi \frac{\varphi^{2}}{M_{\mathrm{P}}^{2}}}} & \text { in prescription I, } \\ \varphi & \text { in prescription II, }\end{cases}
$$

where $M_{\mathrm{P}}=1 / \sqrt{8 \pi G}=2.4 \times 10^{18} \mathrm{GeV}$ is the reduced Planck scale. The prescriptions I and II are claimed to correspond to a $\varphi$-independent ultraviolet (UV) cutoff in the Einstein and Jordan frames, respectively $[13,14]$. Here the Jordan frame refers to the original action with a nonvanishing $\xi$, while the Einstein frame is the one without $\xi$, obtained by the field redefinition of the metric $g_{\mu \nu}$. Note that if we introduce a UV cutoff as a $\varphi$-independent constant in either frame, then it becomes dependent on $\varphi$ in the other frame. If we accept Eq. (1) literally, the value of $\xi$ can be as low as of order 10 in the prescription I $[12,16,17]$ and $10^{2}$ in II $[12,16]$, under the SM criticality. The physical difference comes from the different large $\varphi$ limit of Eq. (1).

In this paper, we will revisit the relation between the UV cutoff and the renormalization scale. We clarify that the different choice of the cutoffs does not directly lead to the difference in Eq. (1). We argue that, in the counterterm formalism, it may be regarded as the choice of the counterterm to cancel the logarithmic divergence, and can be 
absorbed into the choice of the tree-level potential from infinitely many possibilities.

For that purpose, we consider the fermion loop correction to the effective action in a simplified Higgs-Yukawa model. This toy model captures the essential features necessary to grasp what is going on in the realistic Higgs inflation model: As in the real world, we neglect the Higgs mass term which is much smaller than the one from quartic coupling $\lambda_{4}$ at the large field values under consideration; the renormalization group (RG) running of $\lambda_{4}$ is governed by the loop of topquark, which is represented by $\psi .^{2}$

This paper is organized as follows. In Sec. II, we obtain the one-loop effective action in the Higgs-Yukawa model both in the Jordan and Einstein frames. We show that the effective action is independent of the frame if we properly take into account the change of the path integral measure. This change of measure affects only the quartically divergent term, and has nothing to do with the difference between the prescriptions I and II that is related to the logarithmic divergence. In Sec. III, in the counterterm formalism, we show that the difference between prescriptions I and II comes from the choice of the counterterm to cancel the logarithmic divergence. We point out that this difference can be absorbed into the choice of the tree-level potential, including higher dimensional terms, from the infinite possibilities. In Sec. IV, we present a mechanism that uses the gauge kinetic function to stop the running of effective quartic coupling for large $\varphi$ as in the prescription I in Eq. (1), which helps to further flatten the Higgs potential at high scales. In the last section, we summarize our result.

\section{QUANTUM CORRECTION FROM FERMION LOOP}

\section{A. Frames at classical level}

We first review the transformation from Jordan to Einstein frames at the classical level. Our starting action in Jordan frame is

$$
\begin{aligned}
S= & \int \mathrm{d}^{4} x \sqrt{-g}\left[\frac{M_{\mathrm{P}}^{2}}{2} F_{\mathcal{R}}(\varphi) \mathcal{R}-\frac{1}{2} F_{\Phi}(\varphi) g^{\mu \nu} \partial_{\mu} \varphi \partial_{\nu} \varphi-V(\varphi)\right. \\
& \left.-F_{\Psi}(\varphi) \bar{\psi} \gamma^{\mu} D_{\mu} \psi-F_{\mathrm{Y}}(\varphi) y \varphi \bar{\psi} \psi\right],
\end{aligned}
$$

where $y$ is the Yukawa coupling ${ }^{3}$ and $D_{\mu}=\partial_{\mu}+\Omega_{\mu}$ is the general covariant derivative on spinor, with $\Omega_{\mu}$ being the

\footnotetext{
${ }^{2}$ In reality, the loop of gauge bosons also contributes to the running of $\lambda_{4}$. However, the $\varphi$-dependent effective mass of the canonically normalized gauge boson, $g \varphi$, has the same $F_{\mathcal{R}}(\varphi)$ dependence in the Einstein frame, $g \varphi / \sqrt{F_{\mathcal{R}}(\varphi)}$, as the effective mass of fermion $y \varphi$ which becomes $y \varphi / \sqrt{F_{\mathcal{R}}(\varphi)}$; see e.g. Ref. [12]. Therefore the arguments for frame independence and for prescription dependence should apply without modification after we include gauge boson loops.

${ }^{3}$ This $y$ is related to the SM top Yukawa coupling $y_{t}$ by $y=y_{t} / \sqrt{2}$.
}

spin-connection. We assume that we may take a weak-field limit $\varphi \rightarrow 0$ so that we can expand the action around $\varphi=0$. That is,

$$
F_{X}(\varphi)=1+\xi_{X} \frac{\varphi^{2}}{M^{2}}+\cdots
$$

for $X=\mathcal{R}, \Phi, \Psi$ and $\mathrm{Y}$, where $\xi_{X}$ is the first-order nonminimal coupling and $M$ is the typical scale of UV theory, such as the string scale. ${ }^{4}$ We have also assumed for simplicity that the action is invariant under a chiral $Z_{2}$ symmetry

$$
\varphi \rightarrow-\varphi, \quad \psi \rightarrow \gamma_{5} \psi
$$

In general, the potential contains all the higher dimensional terms:

$$
V(\varphi)=\sum_{n: \text { even }, n \geq 0} \lambda_{n} \frac{\varphi^{n}}{M^{n-4}}
$$

By the field redefinition

$$
g_{\mu \nu}^{\mathrm{E}}=F_{\mathcal{R}}(\varphi) g_{\mu \nu},
$$

we obtain the Einstein-frame action ${ }^{5}$

$$
\begin{aligned}
S= & \int \mathrm{d}^{4} x \sqrt{-g_{\mathrm{E}}}\left[\frac{M_{\mathrm{P}}^{2}}{2} \mathcal{R}_{\mathrm{E}}-\frac{1}{2}\left(\frac{F_{\Phi}(\varphi)}{F_{\mathcal{R}}(\varphi)}+\frac{3}{2}\left(\frac{M_{\mathrm{P}} F_{\mathcal{R}}^{\prime}(\varphi)}{F_{\mathcal{R}}(\varphi)}\right)^{2}\right)\right. \\
& \times g_{\mathrm{E}}^{\mu \nu} \partial_{\mu} \varphi \partial_{\nu} \varphi-\frac{V(\varphi)}{\left(F_{\mathcal{R}}(\varphi)\right)^{2}} \\
& \left.-\frac{F_{\Psi}(\varphi)}{\left(F_{\mathcal{R}}(\varphi)\right)^{3 / 2}} \bar{\psi} \gamma_{\mathrm{E}}^{\mu} D_{\mu}^{\mathrm{E}} \psi-\frac{F_{\mathrm{Y}}(\varphi)}{\left(F_{\mathcal{R}}(\varphi)\right)^{2}} y \varphi \bar{\psi} \psi\right] .
\end{aligned}
$$

Here and hereafter, we put either sub- or superscript "E" and "J" on quantities in the Einstein and Jordan frames, respectively, when it is preferable; the ones without such sub- or superscript are given in the Jordan frame unless otherwise stated.

The original Higgs inflation [2] assumes that the potential (5) can be approximated by

$$
V(\varphi)=\lambda_{4} \varphi^{4},
$$

namely $\lambda_{n} \ll 1$ for $n \neq 4$, at around the scale $\varphi \sim M \lesssim M_{\mathrm{P}}{ }^{6}{ }^{6}$ That is, one assumes that all the higher

\footnotetext{
${ }^{4}$ The nonminimal coupling $\xi$ between Higgs and Ricci scalar in the ordinary notation reads $\xi=\xi_{\mathcal{R}} M_{\mathrm{P}}^{2} / M^{2}$ [2]. See also Ref. [18] for more arbitrary extension with large nonminimal couplings.

${ }^{5}$ In Ref. [12], the factor in front of the scalar kinetic term has a typo and should read $\left[\frac{B}{A}+\frac{3}{2} \frac{M_{P}^{2} A^{2}}{A^{2}}\right]$.

${ }^{6}$ For the Higgs inflation under SM criticality $[12,16]$, we further assume the flatness $\lambda_{4} \ll 1$ at high scales. In terms of the quartic coupling $\lambda$ in Refs. [12,16], the quartic coupling in this paper is written as $\lambda_{4}=\lambda / 4$.
} 
order terms are small at $\varphi \sim M$ so that $V \ll M^{4} \lesssim M_{\mathrm{P}}^{4}$. Combined with the assumption $F_{\mathcal{R}}=1+\xi \varphi^{2} / M_{\mathrm{P}}^{2}$, the potential in Eq. (7),

$$
U:=\frac{V}{F_{\mathcal{R}}^{2}},
$$

becomes constant in the large $\varphi$ limit:

$$
U \rightarrow \frac{\lambda_{4}}{\xi^{2}} M_{\mathrm{P}}^{4}
$$

leading to the inflation. The field $\varphi$ is not canonically normalized in the Einstein frame, and the change of equation of motion must be taken into account; see Appendix A. Note that it is important to terminate the expansion of $V$ and $F_{\mathcal{R}}$ at $\varphi^{4}$ and $\varphi_{7}^{2}$, respectively, in order to obtain this constant potential. $^{7}$

\section{B. Frame dependence of UV cutoffs}

Now we take into account the quantum corrections. In this paper, we evaluate the one-loop correction to the effective action from the fermion loop, leaving those from the graviton and $\phi$ loops. $^{8}$ We compute the corrections to $\lambda_{n}$ only. That is, we neglect all the corrections to other couplings $y, \xi_{X}$, etc. and hence do not distinguish the bare and renormalized couplings for them.

In a given frame, short-distance cutoff $\ell$ is given by

$$
\ell^{2}=g_{\mu \nu} \Delta x^{\mu} \Delta x^{\nu} .
$$

Then the metric redefinition (6) relates the cutoff lengths in two frames by

$$
\ell_{\mathrm{J}}^{2}=g_{\mu \nu}^{\mathrm{J}} \Delta x^{\mu} \Delta x^{\nu}=\frac{g_{\mu \nu}^{\mathrm{E}}}{F_{\mathcal{R}}(\varphi)} \Delta x^{\mu} \Delta x^{\nu}=\frac{\ell_{\mathrm{E}}^{2}}{F_{\mathcal{R}}(\varphi)} .
$$

That is, the UV cutoff scales are related by [13]

$$
\Lambda_{\mathrm{E}}^{2}=\frac{\Lambda_{\mathrm{J}}^{2}}{F_{\mathcal{R}}(\varphi)} .
$$

As pointed out in Refs. [13], we may choose either $\Lambda_{\mathrm{J}}$ or $\Lambda_{\mathrm{E}}$ to be independent of $\varphi$, but not both. ${ }^{9}$ In the prescriptions I and II in the original sense [13], we set $\Lambda_{\mathrm{E}}$ and $\Lambda_{\mathrm{J}}$ to be a constant, respectively.

\footnotetext{
${ }^{7}$ The other option is to terminate them at the $2 n$th and $n$th orders, respectively; see the last point in Appendix B.

${ }^{8}$ The correction from $\phi$ loop is proportional to $\lambda_{n}$, which are assumed to be small here; see Refs. $[11,19,20]$ for arguments in support of the smallness of $\lambda_{n}$ at high scales.

${ }^{9}$ We note that such a field-dependent cutoff itself does not lead to any logical inconsistency. For example, a position-dependent momentum cutoff follows from the Pauli-Villars regularization in warped space even if we start from a position-independent bulk mass for the regulator [21]
}

\section{Frame independence of effective action up to quartic divergence}

In general, the effective action should not depend on the choice of frame if we properly take into account the change of the path integral measure as well as that of the cutoff (13). We demonstrate it at the one-loop level under the simplifying assumption given above.

The one-loop effective action induced by the fermion loop in the Jordan frame is given by

$$
\begin{aligned}
e^{i \Delta S_{\text {eff }}^{\mathrm{J}}}: & =\int \mathcal{D}_{g_{\mathrm{J}}} \psi \mathcal{D}_{g_{\mathrm{J}}} \bar{\psi} \\
& \times \exp \left[i \int \mathrm{d}^{4} x \sqrt{-g_{\mathrm{J}}} \bar{\psi}\left(-F_{\Psi} \not_{g_{\mathrm{J}}}-F_{\mathrm{Y}} y \varphi\right) \psi\right] .
\end{aligned}
$$

There is no unique definition of the path integral measure $\mathcal{D}_{g_{\mathrm{J}}} \psi$. Here we take a simple measure that is induced from the following distance in the functional space

$$
\|\delta \psi\|_{g_{\mathrm{J}}}^{2}=\int \mathrm{d}^{4} x \sqrt{-g_{\mathrm{J}}} \delta \bar{\psi} \delta \psi
$$

which is invariant under the diffeomorphism.

Let us rewrite Eq. (14) into the path integral in Einstein frame. Because we have

$$
\begin{aligned}
\|\delta \psi\|_{g_{\mathrm{J}}}^{2} & =\int \mathrm{d}^{4} x \sqrt{-g_{\mathrm{J}}} \delta \bar{\psi} \delta \psi \\
& =\int \mathrm{d}^{4} x \sqrt{-g_{\mathrm{E}}} F_{\mathcal{R}}^{-2} \delta \bar{\psi} \delta \psi,
\end{aligned}
$$

the functional measure satisfies

$$
\begin{aligned}
\mathcal{D}_{g_{\mathrm{J}}} \psi \mathcal{D}_{g_{\mathrm{J}}} \bar{\psi} & =\mathcal{D}_{g_{\mathrm{E}}} \psi \mathcal{D}_{g_{\mathrm{E}}} \bar{\psi}\left(\prod_{x} F_{\mathcal{R}}^{-2}\right)^{-4} \\
& =\mathcal{D}_{g_{\mathrm{E}}} \psi \mathcal{D}_{g_{\mathrm{E}}} \bar{\psi} \exp \left[-4 \operatorname{Tr}_{g_{\mathrm{E}}, \Lambda_{\mathrm{E}}} \ln F_{\mathcal{R}}^{-2}\right],
\end{aligned}
$$

where the measure $\mathcal{D}_{g_{\mathrm{E}}} \psi$ is the one induced from the following distance in the functional space

$$
\|\delta \psi\|_{g_{\mathrm{E}}}^{2}=\int \mathrm{d}^{4} x \sqrt{-g_{\mathrm{E}}} \delta \bar{\psi} \delta \psi
$$

and $\operatorname{Tr}_{g_{\mathrm{E}}, \Lambda_{\mathrm{E}}}$ indicates that the functional trace depends both on the metric $g_{\mathrm{E}}$ and the cutoff $\Lambda_{\mathrm{E}}$; the more explicit form will be presented below. ${ }^{10}$

When we rewrite the functional measure $\mathcal{D}_{g_{\mathrm{J}}} \psi \mathcal{D}_{g_{\mathrm{J}}} \bar{\psi}$ in terms of $\mathcal{D}_{g_{\mathrm{E}}} \psi \mathcal{D}_{g_{\mathrm{E}}} \bar{\psi}$, there has appeared the extra contribution to the effective action:

\footnotetext{
${ }^{10}$ The extra minus sign of -4 in Eq. (17) is from the Jacobian for fermionic variables.
} 


$$
\exp \left[-4 \underset{g_{\mathrm{E}}, \Lambda_{\mathrm{E}}}{\operatorname{Tr}} \ln F_{\mathcal{R}}^{-2}\right]
$$

As we will see below, this factor contains quartic divergence, and is absorbed into the renormalized couplings including the coefficients of higher dimensional terms. Finally, Eq. (14) becomes

$$
\begin{aligned}
e^{i \Delta S_{\text {eff }}^{J}=} & \exp \left[-4 \underset{g_{\mathrm{E}}, \Lambda_{\mathrm{E}}}{\ln } \ln F_{\mathcal{R}}^{-2}\right] \int \mathcal{D}_{g_{\mathrm{E}}} \psi \mathcal{D}_{g_{\mathrm{E}}} \bar{\psi} \\
& \times \exp \left[i \int \mathrm{d}^{4} x \sqrt{-g_{\mathrm{E}}} \bar{\psi}\left(-\frac{F_{\Psi}}{F_{\mathcal{R}}^{3 / 2}} \emptyset_{g_{\mathrm{E}}}-\frac{F_{\mathrm{Y}}}{F_{\mathcal{R}}^{2}} y \varphi\right) \psi\right] \\
= & \exp \left[-4 \underset{g_{\mathrm{E}}, \Lambda_{\mathrm{E}}}{\ln } \ln F_{\mathcal{R}}^{-2}\right] e^{i \Delta S_{\text {eff }}^{\mathrm{E}}},
\end{aligned}
$$

where we have defined the Einstein-frame effective action $\Delta S_{\text {eff }}^{\mathrm{E}}$, which is obtained from the path integral measure $\mathcal{D}_{g_{\mathrm{E}}} \psi \cdot{ }^{11}$ In this section hereafter, we will put the superscript $\mathrm{J}$ (E) for the effective potential of the theory that is defined by using the measure $\mathcal{D}_{g_{\mathrm{J}}} \psi\left(\mathcal{D}_{g_{\mathrm{E}}} \psi\right)$, as well as the effective action.

\section{Explicit computations}

Now we verify the equality (28) through more explicit computations under the assumption that $\varphi$ and $g_{\mu \nu}$ are slowly varying backgrounds so that they may be treated as constants in the computation of the effective action.

As a preparation, let us first compute the extra factor (19) coming from the change of measure:

$$
\begin{aligned}
\exp \left[-4 \underset{g_{\mathrm{E}}, \Lambda_{\mathrm{E}}}{\operatorname{Tr}} \ln F_{\mathcal{R}}^{-2}\right] & =\exp \left[-4 i \int \mathrm{d}^{4} x \sqrt{-g_{\mathrm{E}}}\left\langle x\left|\ln F_{\mathcal{R}}^{-2}\right| x\right\rangle_{\Lambda_{\mathrm{E}}}\right] \\
& =\exp \left[i \int \mathrm{d}^{4} x \sqrt{-g_{\mathrm{E}}}\left(-\frac{\Lambda_{\mathrm{E}}^{4}}{8 \pi^{2}} \ln F_{\mathcal{R}}^{-2}\right)\right],
\end{aligned}
$$

where we have used

$$
\langle x \mid x\rangle_{\Lambda_{\mathrm{E}}}=\int^{\Lambda_{\mathrm{E}}} \frac{\mathrm{d}^{4} p}{(2 \pi)^{4}}=\int_{0}^{\Lambda_{\mathrm{E}}} \frac{2 \pi^{2} p^{3} \mathrm{~d} p}{(2 \pi)^{4}}=\frac{\Lambda_{\mathrm{E}}^{4}}{32 \pi^{2}} .
$$

Here and hereafter, the momentum integral is taken in the Euclidean space.

\footnotetext{
${ }^{11}$ The same argument applies if we start from a different theory defined with another measure induced from the distance (18) instead of Eq. (16). Then the Jordan-frame effective action will receive extra contribution from the change of measure, $\exp \left[4 \operatorname{Tr}_{g_{\mathrm{J}}, \Lambda_{\mathrm{J}}} \ln F_{\mathcal{R}}^{-2}\right]$, which again will make the difference only in the renormalization conditions.
}

The effective action (14) reads

$$
\begin{aligned}
& e^{i \Delta S_{\mathrm{eff}}^{\mathrm{J}}}=\operatorname{Det}_{g_{\mathrm{J}}, \Lambda_{\mathrm{J}}}\left(\frac{-F_{\Psi} \varnothing_{g_{\mathrm{J}}}-F_{\mathrm{Y}} y \varphi}{\mu_{0}}\right) \\
& =\exp \left[\underset{g_{\mathrm{J}}, \Lambda_{\mathrm{J}}}{\operatorname{Tr}} \ln \left(\frac{-F_{\Psi} \not_{g_{\mathrm{J}}}-F_{\mathrm{Y}} y \varphi}{\mu_{0}}\right)\right] \\
& =\exp \left[4 i \int \mathrm{d}^{4} x \sqrt{-g_{\mathrm{J}}} \int^{\Lambda_{\mathrm{J}}} \frac{\mathrm{d}^{4} p}{(2 \pi)^{4}}\right. \\
& \left.\times \frac{1}{2} \ln \left(\frac{F_{\Psi}^{2} p^{2}+F_{\mathrm{Y}}^{2}(y \varphi)^{2}}{\mu_{0}^{2}}\right)\right] \\
& =: \exp \left[i \int \mathrm{d}^{4} x \sqrt{-g_{\mathrm{J}}}\left(-\Delta V_{\mathrm{eff}}^{\mathrm{J}}\right)\right] \text {, }
\end{aligned}
$$

where $\mu_{0}$ is an arbitrary reference scale and we have defined the correction to the Jordan-frame potential $\Delta V_{\text {eff }}^{\mathrm{J}}$. It may be computed as

$$
\begin{aligned}
\Delta V_{\text {eff }}^{\mathrm{J}}= & -2 \int^{\Lambda_{\mathrm{J}}} \frac{\mathrm{d}^{4} p}{(2 \pi)^{4}} \ln \left(\frac{F_{\Psi}^{2} p^{2}+F_{\mathrm{Y}}^{2}(y \varphi)^{2}}{\mu_{0}^{2}}\right) \\
= & -\frac{1}{16 \pi^{2}}\left\{\Lambda_{\mathrm{J}}^{4}\left[\ln \left(F_{\Psi}^{2} \frac{\Lambda_{\mathrm{J}}^{2}+\mathcal{M}_{\mathrm{J}}^{2}}{\mu_{0}^{2}}\right)-\frac{1}{2}\right]\right. \\
& \left.+\Lambda_{\mathrm{J}}^{2} \mathcal{M}_{\mathrm{J}}^{2}+\mathcal{M}_{\mathrm{J}}^{4} \ln \left(\frac{\mathcal{M}_{\mathrm{J}}^{2}}{\Lambda_{\mathrm{J}}^{2}+\mathcal{M}_{\mathrm{J}}^{2}}\right)\right\},
\end{aligned}
$$

where

$$
\mathcal{M}_{\mathrm{J}}(\varphi):=y \varphi \frac{F_{\mathrm{Y}}(\varphi)}{F_{\Psi}(\varphi)}
$$

is the field-dependent mass for canonically normalized fermion in the Jordan frame. ${ }^{12}$ We may rewrite the effective action (23) with the Einstein-frame metric:

$$
\begin{aligned}
e^{i \Delta S_{\text {eff }}^{\mathrm{J}}} & =\exp \left[i \int \mathrm{d}^{4} x \sqrt{-g_{\mathrm{E}}}\left(-\frac{\Delta V_{\text {eff }}^{\mathrm{J}}}{F_{\mathcal{R}}^{2}}\right)\right] \\
& =\exp \left[i \int \mathrm{d}^{4} x \sqrt{-g_{\mathrm{E}}}\left(-\Delta U_{\text {eff }}^{\mathrm{J}}\right)\right],
\end{aligned}
$$

where we have defined the correction to the potential (9). That is,

$$
\begin{aligned}
\Delta U_{\text {eff }}^{\mathrm{J}}= & \frac{\Delta V_{\text {eff }}^{\mathrm{J}}}{F_{\mathcal{R}}^{2}}=-\frac{1}{16 \pi^{2} F_{\mathcal{R}}^{2}}\left\{\Lambda_{\mathrm{J}}^{4}\left[\ln \left(F_{\Psi}^{2} \frac{\Lambda_{\mathrm{J}}^{2}+\mathcal{M}_{\mathrm{J}}^{2}}{\mu_{0}^{2}}\right)-\frac{1}{2}\right]\right. \\
& \left.+\Lambda_{\mathrm{J}}^{2} \mathcal{M}_{\mathrm{J}}^{2}+\mathcal{M}_{\mathrm{J}}^{4} \ln \left(\frac{\mathcal{M}_{\mathrm{J}}^{2}}{\Lambda_{\mathrm{J}}^{2}+\mathcal{M}_{\mathrm{J}}^{2}}\right)\right\}
\end{aligned}
$$

\footnotetext{
${ }^{12}$ For a more realistic top quark loop, $\Delta V_{\text {eff }}$ is multiplied by the color degrees of freedom $N=3$.
} 
To summarize, we have started from the measure (15), and computed the one-loop correction (24). One may worry that the change of path measure (17) might introduce a trace anomaly in addition to Eq. (21). However, it is taken into account as a form of the logarithmic UV cutoff dependence in Eq. (24). Indeed, the constant shift of $\ln \Lambda_{\mathrm{J}}$ correctly reproduces the trace anomaly, as can be seen from Eq. (34) with Eq. (25), compared to Eq. (41).

We may instead perform the field redefinition (6) to the Einstein frame first, and compute the Einstein-frame effective action in the right-hand side of Eq. (28):

$$
\begin{aligned}
e^{i \Delta \Delta_{\text {eff }}^{\mathrm{E}}}= & \left.\exp \left[\underset{g_{\mathrm{E}}, \Lambda_{\mathrm{E}}}{\ln } \ln \frac{-\frac{F_{\Psi}}{F_{\mathcal{R}}^{3 / 2}} \emptyset_{g_{\mathrm{E}}}-\frac{F_{\mathrm{Y}}}{F_{\mathcal{R}}^{2}} y \varphi}{\mu_{0}}\right)\right] \\
= & \exp \left[4 i \int \mathrm{d}^{4} x \sqrt{-g_{\mathrm{E}}} \int^{\Lambda_{\mathrm{E}}} \frac{\mathrm{d}^{4} p}{(2 \pi)^{4}}\right. \\
& \left.\times \frac{1}{2} \ln \left(\frac{\frac{F_{\Psi}^{2}}{F_{\mathcal{R}}^{3}} p^{2}+\frac{F_{\mathrm{Y}}^{2}}{F_{\mathcal{R}}^{4}}(y \varphi)^{2}}{\mu_{0}^{2}}\right)\right] \\
= & \exp \left[i \int \mathrm{d}^{4} x \sqrt{-g_{\mathrm{E}}}\left(-\Delta U_{\mathrm{eff}}^{\mathrm{E}}\right)\right],
\end{aligned}
$$

where $\Delta U_{\text {eff }}^{\mathrm{E}}$ is the fermion loop correction to the potential (9) that is obtained with the measure $\mathcal{D}_{g_{\mathrm{E}}} \psi$ :

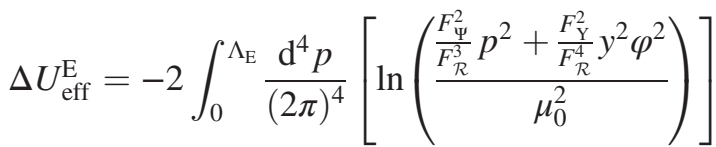

$$
\begin{aligned}
& =-\frac{1}{16 \pi^{2}}\left\{\Lambda_{\mathrm{E}}^{4}\left[\ln \left(\frac{F_{\Psi}^{2}}{F_{\mathcal{R}}^{3}} \frac{\Lambda_{\mathrm{E}}^{2}+\frac{\mathcal{M}_{\mathrm{J}}^{2}}{F_{\mathcal{R}}}}{\mu_{0}^{2}}\right)-\frac{1}{2}\right]\right. \\
& \left.+\Lambda_{\mathrm{E}}^{2} \frac{\mathcal{M}_{\mathrm{J}}^{2}}{F_{\mathcal{R}}}+\frac{\mathcal{M}_{\mathrm{J}}^{4}}{F_{\mathcal{R}}^{2}} \ln \left(\frac{\frac{\mathcal{M}_{\mathrm{J}}^{2}}{F_{\mathcal{R}}}}{\Lambda_{\mathrm{E}}^{2}+\frac{\mathcal{M}_{\mathrm{J}}^{2}}{F_{\mathcal{R}}}}\right)\right\} \text {. }
\end{aligned}
$$

With the identification of UV cutoff scales (13), we may rewrite

$$
\begin{aligned}
\Delta U_{\mathrm{eff}}^{\mathrm{E}}= & -\frac{1}{16 \pi^{2} F_{\mathcal{R}}^{2}}\left\{\Lambda_{\mathrm{J}}^{4}\left[\ln \left(\frac{F_{\Psi}^{2}}{F_{\mathcal{R}}^{4}} \frac{\Lambda_{\mathrm{J}}^{2}+\mathcal{M}_{\mathrm{J}}^{2}}{\mu_{0}^{2}}\right)-\frac{1}{2}\right]\right. \\
& \left.+\Lambda_{\mathrm{J}}^{2} \mathcal{M}_{\mathrm{J}}^{2}+\mathcal{M}_{\mathrm{J}}^{4} \ln \left(\frac{\mathcal{M}_{\mathrm{J}}^{2}}{\Lambda_{\mathrm{J}}^{2}+\mathcal{M}_{\mathrm{J}}^{2}}\right)\right\} .
\end{aligned}
$$

We see that

$$
\Delta U_{\mathrm{eff}}^{\mathrm{E}}=\Delta U_{\mathrm{eff}}^{\mathrm{J}}-\frac{1}{16 \pi^{2}} \Lambda_{\mathrm{E}}^{4} \ln F_{\mathcal{R}}^{-4} .
$$

Using Eq. (21), we see that Eq. (31) is equivalent to Eq. (30). We note that the difference in (31) is quartically divergent, which will be subtracted by the renormalization. In particular, this difference does not change the running of couplings, as we will see.

To summarize, once we fix the path integral measure, say, to be $\mathcal{D}_{g_{\mathrm{J}}} \psi$, we obtain the same result $\Delta U_{\text {eff }}^{\mathrm{J}}$, no matter in which frame we compute it: When we compute it in the Jordan frame, we obtain

$$
\begin{aligned}
e^{i \Delta S_{\mathrm{eff}}^{\mathrm{J}}} & =e^{i \int \mathrm{d}^{4} x \sqrt{-g_{\mathrm{J}}}\left(-\Delta V_{\mathrm{eff}}^{\mathrm{J}}\right)} \\
& =e^{i \int \mathrm{d}^{4} x \sqrt{-g_{\mathrm{E}}}\left(-\Delta U_{\mathrm{eff}}^{\mathrm{J}}\right)},
\end{aligned}
$$

while when we compute it in the Einstein frame,

$$
\begin{aligned}
e^{i \Delta S_{\text {eff }}^{\mathrm{J}}} & =e^{i \int \mathrm{d}^{4} x \sqrt{-g_{\mathrm{E}}}\left(\frac{\Lambda_{\mathrm{E}}^{4}}{8 \pi^{2}} \ln F_{\mathcal{R}}^{-2}\right)} e^{i \int \mathrm{d}^{4} x \sqrt{-g_{\mathrm{E}}}\left(-\left(\Delta U_{\mathrm{eff}}^{\mathrm{J}} \frac{\Lambda_{\mathrm{E}}^{4}}{16 \pi^{2}} \ln F_{\mathcal{R}}^{-4}\right)\right)} \\
& =e^{i \int \mathrm{d}^{4} x \sqrt{-g_{\mathrm{E}}}\left(-\Delta U_{\mathrm{eff}}^{\mathrm{J}}\right)} .
\end{aligned}
$$

We have explicitly checked that these two agree.

The frame independence of the effective potential has been verified in various ways: In Refs. [22,23], the authors have obtained one-loop RG equations for the tree level action in both the Jordan and Einstein frames, and have found the agreement between both results; see also appendix of Ref. [24]. In Ref. [25], the authors checked that both the tree-level actions are equivalent when written in terms of dimensionless variables, as it should be. In Ref. [26], the authors have computed the one-loop divergent part of the effective potential in both frames, and have shown that both coincide at on-shell. In Refs. [27-29], the authors have discussed frame independence of physical observables.

\section{PRESCRIPTIONS I AND II}

We now discuss the meaning of the prescriptions I and II. We first clarify how the difference of the prescriptions in Eq. (1) arises in the ordinary context. Then we will show, in the counterterm formalism, that this difference can be absorbed into the choice from infinitely many possibilities of the coefficients of higher dimensional terms in the treelevel potential.

We consider the cutoff theory containing infinite number of higher dimensional terms. We tune the infinite number of bare couplings in the large cutoff limit $\Lambda_{\mathrm{J}}, \Lambda_{\mathrm{E}} \rightarrow \infty$ such that the renormalized effective potential becomes a function of $\varphi / M$, where $M$ is the physical mass scale; see, e.g. Ref. [30]. We work in the counterterm formalism so that $\mathcal{M}_{\mathrm{J}}$ and $F_{\Psi}$ are treated as finite renormalized quantities. To be concrete, we consider the theory defined by the path integral measure $\mathcal{D}_{g_{\mathrm{J}}} \psi$, and we omit the superscript $\mathrm{J}$ from the potentials $V^{\mathrm{J}}, \Delta V_{\text {eff }}^{\mathrm{J}}, \ldots$, etc. hereafter. ${ }^{13}$

\footnotetext{
${ }^{13}$ Exactly the same argument applies if we consider the theory defined by the measure $\mathcal{D}_{g_{\mathrm{E}}} \psi$.
} 


\section{A. Prescription II in ordinary context}

We clarify how the prescription II in Eq. (1) appears in the ordinary context. The contribution from fermion to the effective potential (24) contains the quartic, quadratic, and logarithmic divergences:

$$
\begin{aligned}
\Delta V_{\text {eff }}= & -\frac{1}{16 \pi^{2}}\left\{\Lambda_{\mathrm{J}}^{4}\left[\ln \left(F_{\Psi}^{2} \frac{\Lambda_{\mathrm{J}}^{2}}{\mu_{0}^{2}}\right)-\frac{1}{2}\right]+2 \Lambda_{\mathrm{J}}^{2} \mathcal{M}_{\mathrm{J}}^{2}\right. \\
& \left.+\mathcal{M}_{\mathrm{J}}^{4}\left(\ln \frac{\mathcal{M}_{\mathrm{J}}^{2}}{\Lambda_{\mathrm{J}}^{2}}-\frac{1}{2}\right)\right\}+\mathcal{O}\left(\Lambda_{\mathrm{J}}^{-2}\right) .
\end{aligned}
$$

Then in the full effective potential

$$
V=V_{\mathrm{B}}+\Delta V_{\mathrm{eff}},
$$

we cancel the divergences in Eq. (34) by the bare couplings $\lambda_{n \mathrm{~B}}$ in the bare potential

$$
V_{\mathrm{B}}:=\sum_{n} \lambda_{n \mathrm{~B}} \frac{\varphi^{n}}{M^{n-4}} .
$$

The quartic and quadratic divergences in Eq. (34) can be simply subtracted by the counterterm

$V_{\text {power }}^{\text {c.t. }}=\frac{1}{16 \pi^{2}}\left\{\Lambda_{\mathrm{J}}^{4}\left[\ln \left(F_{\Psi}^{2} \frac{\Lambda_{\mathrm{J}}^{2}}{\mu_{0}^{2}}\right)-\frac{1}{2}\right]+2 \Lambda_{\mathrm{J}}^{2} \mathcal{M}_{\mathrm{J}}^{2}\right\}$.

However, we need a special care in subtracting the logarithmic divergence in Eq. (34) because the counter term should be analytic around $\varphi=0$ : A counterterm having $\ln \mathcal{M}_{\mathrm{J}}=\ln \varphi+\cdots$ breaks the analyticity around $\varphi=0$. In particular, the $(n+1)$ th derivative of the term $\varphi^{n} \ln \varphi$ is singular, and the $(n+1)$-point function becomes ill-defined in the weak field limit $\varphi \rightarrow 0$ if the bare action has such a term. ${ }^{14}$ Because we employ the analytic tree-level potential, the counterterm should then be analytic too.

A natural choice of the counterterm that is analytic around $\varphi=0$ would be

$$
V_{\log }^{\text {c.t.I }}=\frac{\mathcal{M}_{\mathrm{J}}^{4}}{16 \pi^{2}} \ln \frac{\mu^{2}}{\Lambda_{\mathrm{J}}^{2}},
$$

where $\mu$ is the renormalization scale. The resultant bare potential is

$$
\begin{aligned}
V_{\mathrm{B}}^{\mathrm{II}}= & V_{\mathrm{R}}^{\mathrm{II}}+V_{\text {power }}^{\text {c.t. }}+V_{\log }^{\text {c.t.I }} \\
= & V_{\mathrm{R}}^{\mathrm{II}}+\frac{1}{16 \pi^{2}}\left\{\Lambda_{\mathrm{J}}^{4}\left[\ln \left(F_{\Psi}^{2} \frac{\Lambda_{\mathrm{J}}^{2}}{\mu_{0}^{2}}\right)-\frac{1}{2}\right]\right. \\
& \left.+2 \Lambda_{\mathrm{J}}^{2} \mathcal{M}_{\mathrm{J}}^{2}+\mathcal{M}_{\mathrm{J}}^{4} \ln \frac{\mu^{2}}{\Lambda_{\mathrm{J}}^{2}}\right\}
\end{aligned}
$$

\footnotetext{
${ }^{14}$ In contrast, the singular behavior of $(n+1)$ th derivative of the effective action represents the infrared singularity of the $(n+1)$-point scattering of massless scalar, which is cured by taking into account the Higgs mass in reality and/or by concentrating on the infrared-safe physical quantities.
}

where $V_{\mathrm{R}}^{\mathrm{II}}$ is the tree-level potential in the counterterm formalism. We note that $V_{\mathrm{R}}^{\mathrm{II}}$ is $\mu$-dependent:

$$
V_{\mathrm{R}}^{\mathrm{II}}(\varphi, \mu)=\sum_{n} \lambda_{n \mathrm{R}}(\mu) \frac{\varphi^{n}}{M^{n-4}},
$$

and the $\mu$-independence of $V_{\mathrm{B}}$ determines the running of $\lambda_{n \mathrm{R}}(\mu)$ via Eq. (39). In particular, because $\mathcal{M}_{\mathrm{J}}=y \varphi+\mathcal{O}\left(\varphi^{3}\right)$, we obtain the ordinary running of the quartic coupling:

$$
\frac{\mathrm{d} \lambda_{4 \mathrm{R}}(\mu)}{\mathrm{d} \ln \mu}=-\frac{y^{4}}{8 \pi^{2}} \text {. }
$$

Substituting the bare potential (39) into $V_{\mathrm{B}}$ in Eq. (35), we obtain

$$
\begin{aligned}
V(\varphi) & =V_{\mathrm{B}}^{\mathrm{II}}+\Delta V_{\mathrm{eff}} \\
& =V_{\mathrm{R}}^{\mathrm{II}}(\varphi, \mu)+\Delta V_{\mathrm{R}}^{\mathrm{II}}(\varphi, \mu),
\end{aligned}
$$

where

$$
\Delta V_{\mathrm{R}}^{\mathrm{II}}(\varphi, \mu):=-\frac{\left[\mathcal{M}_{\mathrm{J}}(\varphi)\right]^{4}}{16 \pi^{2}}\left(\ln \frac{\left[\mathcal{M}_{\mathrm{J}}(\varphi)\right]^{2}}{\mu^{2}}-\frac{1}{2}\right)
$$

is the one-loop correction in the counterterm formalism in the prescription II. Now both $V_{\mathrm{R}}^{\mathrm{II}}$ and $\Delta V_{\mathrm{R}}^{\mathrm{II}}$ are finite.

When we want to minimize the correction (43), we may choose the renormalization scale ${ }^{15}$

$$
\mu \sim \mathcal{M}_{\mathrm{J}} .
$$

This result reproduces the prescription II in the sense of Eq. (1), namely $\mu \sim \varphi$ for $F_{\Psi}=F_{\mathrm{Y}}=1$.

\section{B. Prescription $I$ in ordinary context}

We clarify how the prescription I in Eq. (1) appears in the ordinary context. We can rewrite Eq. (34) by using Eq. $(13)^{16}$ :

$$
\begin{aligned}
\Delta V_{\text {eff }}= & -\frac{F_{\mathcal{R}}^{2}}{16 \pi^{2}}\left\{\Lambda_{\mathrm{E}}^{4}\left[\ln \left(F_{\Psi}^{2} \frac{F_{\mathcal{R}} \Lambda_{\mathrm{E}}^{2}}{\mu_{0}^{2}}\right)-\frac{1}{2}\right]\right. \\
& \left.+2 \Lambda_{\mathrm{E}}^{2} \frac{\mathcal{M}_{\mathrm{J}}^{2}}{F_{\mathcal{R}}}+\frac{\mathcal{M}_{\mathrm{J}}^{4}}{F_{\mathcal{R}}^{2}}\left(\ln \frac{\mathcal{M}_{\mathrm{J}}^{2} / F_{\mathcal{R}}}{\Lambda_{\mathrm{E}}^{2}}-\frac{1}{2}\right)\right\}+\mathcal{O}\left(\Lambda_{\mathrm{E}}^{-2}\right) .
\end{aligned}
$$

The quartic and quadratic divergences are canceled by the same counterterm (37). This time, a natural choice to cancel the logarithmic divergence would be, instead of Eq. (38),

\footnotetext{
${ }^{15}$ The constant $-1 / 2$ is scheme-dependent and does not affect our argument here.

${ }^{16}$ This may also be verified by substituting Eq. (29) into Eq. (31), dividing both-hand sides by $F_{\mathcal{R}}^{2}$, and expanding it in terms of $\Lambda_{\mathrm{E}}$.
} 


$$
V_{\log }^{\text {c.t.I }}=\frac{\mathcal{M}_{\mathrm{J}}^{4}}{16 \pi^{2}} \ln \frac{\mu^{2}}{\Lambda_{\mathrm{E}}^{2}} .
$$

Then the bare potential becomes

$$
\begin{aligned}
V_{\mathrm{B}}^{\mathrm{I}}= & V_{\mathrm{R}}^{\mathrm{I}}+V_{\text {power }}^{\text {c.t. }}+V_{\log }^{\text {c.t.I }} \\
= & V_{\mathrm{R}}^{\mathrm{I}}+\frac{1}{16 \pi^{2}}\left\{\Lambda_{\mathrm{E}}^{4} F_{\mathcal{R}}^{2}\left[\ln \left(F_{\Psi}^{2} \frac{F_{\mathcal{R}} \Lambda_{\mathrm{E}}^{2}}{\mu_{0}^{2}}\right)-\frac{1}{2}\right]\right. \\
& \left.+2 \Lambda_{\mathrm{E}}^{2} F_{\mathcal{R}} \mathcal{M}_{\mathrm{J}}^{2}+\mathcal{M}_{\mathrm{J}}^{4} \ln \frac{\mu^{2}}{\Lambda_{\mathrm{E}}^{2}}\right\},
\end{aligned}
$$

and we obtain

$$
\begin{aligned}
V(\varphi) & =V_{\mathrm{B}}^{\mathrm{I}}+\Delta V_{\mathrm{eff}} \\
& =V_{\mathrm{R}}^{\mathrm{I}}(\varphi, \mu)+\Delta V_{\mathrm{R}}^{\mathrm{I}}(\varphi, \mu),
\end{aligned}
$$

where

$\Delta V_{\mathrm{R}}^{\mathrm{I}}(\varphi, \mu):=-\frac{\left[\mathcal{M}_{\mathrm{J}}(\varphi)\right]^{4}}{16 \pi^{2}}\left(\ln \frac{\left[\mathcal{M}_{\mathrm{J}}(\varphi)\right]^{2} / F_{\mathcal{R}}}{\mu^{2}}-\frac{1}{2}\right)$.

Again the $\mu$-independence of $V$ fixes the running of the couplings. The running of quartic coupling becomes the same as in Eq. (41) because $F_{\mathcal{R}}=1+\mathcal{O}\left(\varphi^{2}\right)$ and hence the $\varphi^{4}$ term is not affected by $\ln F_{\mathcal{R}}$; see the discussion below.

When we want to minimize the second term in Eq. (48), we may choose the renormalization scale

$$
\mu \sim \frac{\mathcal{M}_{\mathrm{J}}}{\sqrt{F_{\mathcal{R}}}}
$$

This result reproduces the prescription $\mathrm{I}$ in the sense of Eq. (1), namely $\mu \sim \varphi / \sqrt{1+\xi \varphi^{2} / M_{\mathrm{P}}^{2}}$ for $F_{\Psi}=F_{\mathrm{Y}}=1$; see also footnote 4 .

\section{Where the difference comes from}

Let us summarize the difference between the prescriptions I and II. The difference of prescriptions I and II comes from that of the subtractions of logarithmic divergence in Eqs. (38) and (46):

$V_{\log }^{\text {c.t.I }}-V_{\log }^{\text {c.t.II }}=\frac{\mathcal{M}_{\mathrm{J}}^{4}}{16 \pi^{2}} \ln \frac{\Lambda_{\mathrm{J}}^{2}}{\Lambda_{\mathrm{E}}^{2}}=\frac{\mathcal{M}_{\mathrm{J}}^{4}}{16 \pi^{2}} \ln F_{\mathcal{R}}=\frac{\xi_{\mathcal{R}} y^{4}}{16 \pi^{2}} \frac{\varphi^{6}}{M^{2}}+\cdots$,

where we used Eq. (13). This difference amounts to the finite renormalization of $V_{\mathrm{R}}$. Note that the difference (51) is analytic around $\varphi=0$ and that it has only higher order terms with $n \geq 6$.

Originally the prescription has been introduced as a choice of frame in which the theory is defined, and it was believed that the radiative correction to the effective potential is minimized by the choice of the renormalization scale as in Eq. (1). As we have shown, however, the physics does not depend on the choice of the frame in which the theory is defined. Instead, for a given renormalized treelevel action, the difference of the prescriptions (1) can be understood as that of the logarithmic counterterms (51): The different counterterms lead to the different scales (44) and (50) that minimize the radiative corrections.

\section{Renormalized potential}

Theoretically, the potential $V_{\mathrm{R}}$ in Eq. (42) or (48),

$$
V_{\mathrm{R}}=\sum_{n: \text { even, } n \geq 0} \lambda_{n \mathrm{R}}(\mu) \frac{\varphi^{n}}{M^{n-4}},
$$

may take arbitrary form, so long as it is analytic around $\varphi=0$. How do we determine its form?

We may reproduce the ordinary Higgs inflation [2] that does not assume the criticality, by tuning the infinite number of bare couplings such that $V_{\mathrm{R}}$ becomes

$$
\left.V_{\mathrm{R}}(\varphi, \mu)\right|_{\mu \sim M} \simeq \lambda_{4 \mathrm{R}}(\mu) \varphi^{4},
$$

where all the couplings $\lambda_{n \mathrm{R}}(\mu)$ with $n \neq 4$ are suppressed at $\mu=M$. When the form (53) is put into Eqs. (42) and (48), which result from the counterterms (38) and (46), we obtain the Higgs potential in the prescriptions II and I in the ordinary context, respectively:

$$
\begin{aligned}
V^{\mathrm{II}} & =\lambda_{4 \mathrm{R}}(\mu) \varphi^{4}-\frac{\mathcal{M}_{\mathrm{J}}^{4}}{16 \pi^{2}}\left(\ln \frac{\mathcal{M}_{\mathrm{J}}^{2}}{\mu^{2}}-\frac{1}{2}\right), \\
V^{\mathrm{I}} & =\lambda_{4 \mathrm{R}}(\mu) \varphi^{4}-\frac{\mathcal{M}_{\mathrm{J}}^{4}}{16 \pi^{2}}\left(\ln \frac{\mathcal{M}_{\mathrm{J}}^{2} / F_{\mathcal{R}}}{\mu^{2}}-\frac{1}{2}\right) .
\end{aligned}
$$

However, we may as well obtain the potential of the form of $V^{\mathrm{I}}$ in Eq. (55) even when we employ the counterterm $V_{\log }^{\text {c.t.II }}$ in Eq. (38) if we choose the following form of the tree-level potential $V_{\mathrm{R}}$ in Eq. (42),

$$
V_{\mathrm{R}}(\varphi, \mu)=\lambda_{4 \mathrm{R}}(\mu) \varphi^{4}+\frac{\left[\mathcal{M}_{\mathrm{J}}(\varphi)\right]^{4}}{16 \pi^{2}} \ln F_{\mathcal{R}}(\varphi),
$$

instead of the form (53). From the same counterterm $V_{\log }^{\text {c.t.II in }}$ Eq. (38), we may obtain the forms (54) and (55) by assuming the tree-level potentials (53) and (56), respectively. Recall that the second term in the right-hand side of Eq. (56) modifies only the higher dimensional terms of $\mathcal{O}\left(\varphi^{6}\right)$. There are infinitely many possibilities for the tree-level potential. Therefore there is no reason to suppose one (or any) of the above two as the proper tree-level potential if we restrict ourselves to the low-energy effective field theory. ${ }^{17}$

\footnotetext{
${ }^{17}$ The tree-level potential is determined once we fix the underlying UV-finite theory; see, e.g., Refs. [19,20] for discussions based on string theory.
} 


\section{FLATTENING HIGGS POTENTIAL BY GAUGE KINETIC FUNCTION}

Let us turn to more realistic running of the quartic coupling in the SM:

$$
V_{\mathrm{SM}}=\lambda_{4 \mathrm{R}}(\mu) \varphi^{4}+\Delta V_{\mathrm{R}}(\varphi, \mu),
$$

where $\Delta V_{\mathrm{R}}$ is the finite correction (43) or (49) in the counterterm formalism; see also footnote 6 . In the SM, $\beta_{4 \mathrm{R}}:=\frac{\mathrm{d}}{\mathrm{d} \ln \mu} \lambda_{4 \mathrm{R}}$ turns from negative to positive around the scale $\mu_{\min } \sim 10^{17} \mathrm{GeV}$, and we may approximate as $[12,16]$

$$
\lambda_{4 \mathrm{R}}(\mu) \approx \lambda_{4 \mathrm{R}}^{\min }+b_{4 \mathrm{R}}\left(\ln \frac{\mu}{\mu_{\min }}\right)^{2}
$$

where $b_{4 \mathrm{R}}$ can be computed within the $\mathrm{SM}$ as

$$
b_{4 \mathrm{R}} \simeq \frac{0.1}{\left(16 \pi^{2}\right)^{2}} \simeq 5 \times 10^{-6} .
$$

The negative $\beta_{4 \mathrm{R}}$ for $\mu<\mu_{\min }$ is dominated by top quark loop, while the positive $\beta_{4 \mathrm{R}}$ for $\mu>\mu_{\min }$ by the $U(1)_{Y}$ and $S U(2)_{L}$ gauge boson loops.

For top quark loop, the contribution is through the effective mass $\mathcal{M}_{\mathrm{J}}=y \varphi F_{\mathrm{Y}} / F_{\Psi}$. In the prescription I in the sense of Eq. (1), namely in Eq. (55) with the tree-level potential (53), we get the constant $\mu$ in the large $\varphi$ limit,

$$
\mu \sim \frac{\mathcal{M}_{\mathrm{J}}}{\sqrt{F_{\mathcal{R}}}} \rightarrow \frac{M_{\mathrm{P}}}{\sqrt{\xi}},
$$

and the effective quartic coupling $\left.\lambda_{4 \mathrm{R}}(\mu)\right|_{\mu \sim \mathcal{M}_{\mathrm{J}} / \sqrt{F_{\mathcal{R}}}}$ stops running for large $\varphi$ [15]. ${ }^{18}$ This mechanism makes the potential even flatter at the SM criticality and helps to earn a sufficiently large $e$-folding number for smaller $\xi \sim 10$; in the prescription II we lack this mechanism and need larger $\xi \sim 10^{2}[12,16]$.

Similarly, the contribution of the gauge boson loop is through [12]

$$
\mathcal{M}_{\mathrm{J}}^{\text {gauge }}=g \varphi \sqrt{\frac{F_{\Phi}}{F_{\mathrm{g}}}}
$$

where $g$ is the gauge coupling and $F_{\mathrm{g}}$ is the gauge kinetic function, namely the function of $\varphi$ in front of the gauge kinetic term.

\footnotetext{
${ }^{18}$ Here we have assumed $F_{\mathrm{Y}}=F_{\Psi}=1$. If $F_{\mathrm{Y}}=1+\xi_{\mathrm{Y}} \varphi^{2} / M^{2}$ and $F_{\Psi}=1+\xi_{\Psi} \varphi^{2} / M^{2}$, we obtain

$$
\mu \sim \frac{\mathcal{M}_{\mathrm{J}}}{\sqrt{F_{\mathcal{R}}}} \rightarrow \frac{\xi_{\mathrm{Y}}}{\sqrt{\xi} \xi_{\Psi}} M_{\mathrm{P}}
$$
}

instead of Eq. (60); see also footnote 4.
When we raise the scale beyond $\mu>\mu_{\min }$ in the SM, the top Yukawa coupling becomes smaller and smaller. To the first approximation, the running at $\mu>\mu_{\min }$ is governed by the gauge boson loop. Then in the prescription II in the ordinary context, which corresponds to Eq. (54) with the tree-level potential (53), the effective potential becomes

$$
V_{\mathrm{SM}}=\left.\lambda_{4 \mathrm{R}}(\mu) \varphi^{4}\right|_{\mu=\mathcal{M}_{\mathrm{J}}^{\text {gauge }}}
$$

When we assume that $F_{\Phi} \simeq 1$, we obtain

$$
\mathcal{M}_{\mathrm{J}}^{\text {gauge }}=\frac{g \varphi}{\sqrt{1+\xi_{\mathrm{g}} \frac{\varphi^{2}}{M^{2}}}} .
$$

In the large- $\varphi$ limit,

$$
\mathcal{M}_{\mathrm{J}}^{\text {gauge }} \rightarrow \frac{g}{\sqrt{\xi_{\mathrm{g}}}} M
$$

We propose that this can be used in the prescription II in the ordinary context as an alternative mechanism to Eq. (60) in order to stop the running of quartic coupling $\left.\lambda_{4 \mathrm{R}}(\mu)\right|_{\mu \sim \mathcal{M}_{\mathrm{J}}^{\text {gauge }}}$ for large $\varphi{ }^{19}$

The $\varphi$-dependent mass (63) takes the same form as the prescription I in Eq. (1) if we neglect the gauge coupling $g$. Therefore, for example, we may set $\xi_{\mathrm{g}}=\xi_{\mathcal{R}}$ and $M=M_{\mathrm{P}}$, then the subsequent analysis becomes identical to those in Ref. [12]. This serves as an explicit example of viable parameter set that realizes the above-mentioned idea.

\section{SUMMARY}

We have analyzed the one-loop effective action in the simplified Higgs-Yukawa model, which captures essential features of the Higgs potential in the Higgs inflation. We have shown that the effective actions obtained in the Jordan and Einstein frames are exactly the same if we properly take into account the change of path integral measure. We show that, in the counterterm formalism, the prescriptions I and II are merely two specific choices of counterterms to cancel the logarithmic divergence. We point out that the difference between I and II can be absorbed into the choice

\footnotetext{
${ }^{19}$ When the top Yukawa contribution is non-negligible, one may further introduce e.g.

$$
F_{\Psi}(\varphi)=\sqrt{1+2 \xi_{\Psi} \frac{\varphi^{2}}{M^{2}}}=1+\xi_{\Psi} \frac{\varphi^{2}}{M^{2}}-\frac{\xi_{\Psi}^{2}}{2} \frac{\varphi^{4}}{M^{4}}+\frac{\xi_{\Psi}^{3}}{2} \frac{\varphi^{6}}{M^{6}}+\cdots
$$
}

together with $F_{\mathrm{Y}}=1$, which stops running due to the top contribution too:

$$
\mathcal{M}_{\mathrm{J}}=\frac{y \varphi}{\sqrt{1+2 \xi_{\Psi} \frac{\varphi^{2}}{M^{2}}}} \rightarrow \frac{y}{\sqrt{2 \xi_{\Psi}}} M .
$$


of tree-level potential, including higher dimensional terms, from infinitely many possibilities.

We have also proposed a mechanism to stop the running of the effective quartic coupling in the prescription II in the ordinary context, using the gauge kinetic function: $\mu \sim \varphi / \sqrt{F_{\mathrm{g}}} \rightarrow M / \sqrt{\xi_{\mathrm{g}}}$. Detailed phenomenological study of this scenario will be presented in a separate publication.

We briefly comment on the remaining points to be addressed: In this paper, we have concentrated on the fermion loop. It would be worth including the scalar loop, as in Ref. [26], and also the gauge boson loop. It is also worth studying the issue of gauge dependence in these loops.

\section{ACKNOWLEDGMENTS}

We thank Seong Chan Park, Mikhail Shaposhnikov, and Satoshi Yamaguchi for useful discussions. The work of Y.H. and K. O. are supported in part by JSPS KAKENHI Grant Nos. 16J06151 and 23104009, 15K05053, respectively.

\section{APPENDIX A: CLASSICAL DYNAMICS IN TERMS OF JORDAN-FRAME FIELD}

When we consider the classical dynamics of scalar field under gravity, it is convenient to define the canonically normalized scalar field

$$
\mathrm{d} \chi=\mathcal{G}(\varphi) \mathrm{d} \varphi,
$$

where

$$
\mathcal{G}(\varphi):=\sqrt{\frac{F_{\Phi}(\varphi)}{F_{\mathcal{R}}(\varphi)}+\frac{3}{2}\left(\frac{M_{\mathrm{P}} F_{\mathcal{R}}^{\prime}(\varphi)}{F_{\mathcal{R}}(\varphi)}\right)^{2}} .
$$

We use the Friedmann-Lemaître-Robertson-Walker ansatz ${ }^{20}$

$$
g_{\mu \nu}^{\mathrm{E}} \mathrm{d} x^{\mu} \mathrm{d} x^{\nu}=-\mathrm{d} t_{\mathrm{E}}^{2}+\left(a_{\mathrm{E}}\left(t_{\mathrm{E}}\right)\right)^{2} \mathrm{~d} x^{2} .
$$

In the Einstein frame, the Einstein equations reduce to the ordinary Friedmann equation ${ }^{21}$ :

$$
\begin{aligned}
H_{\mathrm{E}}^{2} & =\frac{\rho_{\mathrm{E}}}{3 M_{\mathrm{P}}^{2}}, \\
\frac{\mathrm{d} \rho_{\mathrm{E}}}{\mathrm{d} t_{\mathrm{E}}} & =-3\left(\rho_{\mathrm{E}}+p_{\mathrm{E}}\right) H_{\mathrm{E}},
\end{aligned}
$$

where

$$
\rho_{\mathrm{E}}=\frac{1}{2}\left(\frac{\mathrm{d} \chi}{\mathrm{d} t_{\mathrm{E}}}\right)^{2}+U_{\mathrm{E}}(\varphi)
$$

\footnotetext{
${ }^{20} \mathrm{We}$ neglect the spatial curvature, but may recover it by $\mathrm{d} \boldsymbol{x}^{2} \rightarrow \mathrm{d} \boldsymbol{x}^{2}+K \frac{(\boldsymbol{x} \cdot \mathrm{d} \boldsymbol{x})^{2}}{1-K x^{2}}$.

${ }^{21}$ We may recover the spatial curvature by $H_{\mathrm{E}}^{2} \rightarrow H_{\mathrm{E}}^{2}+\frac{K}{a^{2}}$.
}

$$
p_{\mathrm{E}}=\frac{1}{2}\left(\frac{\mathrm{d} \chi}{\mathrm{d} t_{\mathrm{E}}}\right)^{2}-U_{\mathrm{E}}(\varphi)
$$

and $H_{\mathrm{E}}:=\frac{1}{a_{\mathrm{E}}} \frac{\mathrm{d} a_{\mathrm{E}}}{\mathrm{d} t_{\mathrm{E}}}$.

The Higgs-field equation reads

$$
\frac{\mathrm{d}^{2} \chi}{\mathrm{d} t_{\mathrm{E}}^{2}}+3 H_{\mathrm{E}} \frac{\mathrm{d} \chi}{\mathrm{d} t_{\mathrm{E}}}=-\frac{\mathrm{d} U_{\mathrm{E}}}{\mathrm{d} \chi}
$$

In terms of the Jordan-frame field,

$$
\frac{\mathrm{d}^{2} \varphi}{\mathrm{d} t_{\mathrm{E}}^{2}}+\frac{\mathrm{d} \varphi}{\mathrm{d} t_{\mathrm{E}}}\left(3 H_{\mathrm{E}}+\frac{\mathrm{d}}{\mathrm{d} t_{\mathrm{E}}} \ln \mathcal{G}\right)=-\frac{1}{\mathcal{G}^{2}} \frac{\mathrm{d} U_{\mathrm{E}}}{\mathrm{d} \varphi}
$$

The universe expands with the rate $H_{\mathrm{E}}$, whereas the Jordanframe field $\varphi$ receives extra friction $\frac{\mathrm{d}}{\mathrm{d} t_{\mathrm{E}}} \ln \mathcal{G}$, and rolls slower (faster) than under its absence when it is positive (negative). This term will turn out to be the same order as the slow-roll parameter under the slow-roll condition shown below.

We assume the slow-roll inflation. The slow-roll parameters read

$$
\begin{gathered}
\epsilon:=\frac{M_{\mathrm{P}}^{2}}{2 U_{\mathrm{E}}^{2}}\left(\frac{\mathrm{d} U_{\mathrm{E}}}{\mathrm{d} \chi}\right)^{2}=\frac{M_{\mathrm{P}}^{2}}{2 U_{\mathrm{E}}^{2} \mathcal{G}^{2}}\left(\frac{\mathrm{d} U_{\mathrm{E}}}{\mathrm{d} \varphi}\right)^{2} \ll 1, \\
\eta:=\frac{M_{\mathrm{P}}^{2}}{U_{\mathrm{E}}} \frac{\mathrm{d}^{2} U_{\mathrm{E}}}{\mathrm{d} \chi^{2}}=\frac{M_{\mathrm{P}}^{2}}{U_{\mathrm{E}} \mathcal{G}} \frac{\mathrm{d}}{\mathrm{d} \varphi}\left(\frac{1}{\mathcal{G}} \frac{\mathrm{d} U_{\mathrm{E}}}{\mathrm{d} \varphi}\right) \ll 1 .
\end{gathered}
$$

The Friedmann and Higgs-field equations become, respectively,

$$
\begin{aligned}
& 3 M_{\mathrm{P}}^{2} H_{\mathrm{E}}^{2}=U_{\mathrm{E}}, \\
& 3 H_{\mathrm{E}} \frac{\mathrm{d} \chi}{\mathrm{d} t_{\mathrm{E}}}=-\frac{\mathrm{d} U_{\mathrm{E}}}{\mathrm{d} \chi} .
\end{aligned}
$$

In terms of the Jordan-frame field, the latter reads

$$
3 H_{\mathrm{E}} \frac{\mathrm{d} \varphi}{\mathrm{d} t_{\mathrm{E}}}=-\frac{1}{\mathcal{G}^{2}} \frac{\mathrm{d} U_{\mathrm{E}}}{\mathrm{d} \varphi}=:-\frac{\mathrm{d} \mathcal{U}_{\mathrm{E}}}{\mathrm{d} \varphi},
$$

where we have defined the effectual potential

$$
\mathcal{U}_{\mathrm{E}}=\int \mathrm{d} \varphi \frac{1}{\mathcal{G}^{2}} \frac{\mathrm{d} U_{\mathrm{E}}}{\mathrm{d} \varphi}+\text { const }
$$

which takes into account the effect from $\mathcal{G}$. Using this potential, the slow-roll parameters can be rewritten as

$$
\begin{aligned}
\epsilon & =\frac{\mathcal{G}^{2} M_{\mathrm{P}}^{2}}{2 U_{\mathrm{E}}^{2}}\left(\frac{\mathrm{d} \mathcal{U}_{\mathrm{E}}}{\mathrm{d} \varphi}\right)^{2}, \\
\eta & =\frac{M_{\mathrm{P}}^{2}}{U_{\mathrm{E}}}\left(\frac{\mathrm{d}^{2} \mathcal{U}_{\mathrm{E}}}{\mathrm{d} \varphi^{2}}+\frac{\mathrm{d} \mathcal{U}_{\mathrm{E}}}{\mathrm{d} \varphi} \frac{\mathrm{d}}{\mathrm{d} \varphi} \ln \mathcal{G}\right),
\end{aligned}
$$


though this expression may not be particularly convenient. In this paper, we omit the subscript $\mathrm{E}$ from $U_{\mathrm{E}}$ and $\mathcal{U}_{\mathrm{E}}$ which are always given in the Einstein frame.

\section{APPENDIX B: EXAMPLES THAT LEAD TO VARIOUS HIGGS INFLATIONS}

Even if we decide to take the simple form of the treelevel potential (53) in Eq. (42) or (48), we still have freedom to choose any form of $F_{X}(\varphi)$ s. We review several examples that lead to viable cosmic inflations:

(i) In Ref. [12], the authors have spelled out the result from prescriptions I and II in the ordinary context, with the tree-level potential (53) in Eq. (48) and (42), respectively, and with the function $F_{X}=1$ except for $F_{\mathcal{R}}=1+\xi_{\mathcal{R}} \frac{\varphi^{2}}{M^{2}}$. The former prescription I allows smaller $\xi:=\xi_{\mathcal{R}} M_{\mathrm{P}}^{2} / M^{2} \sim 10$ because the coupling stops running for $\varphi \gg M / \sqrt{\xi_{\mathcal{R}}}$ :

$$
\mu \sim \frac{\varphi}{\sqrt{F_{\mathcal{R}}}} \rightarrow \frac{M}{\sqrt{\xi_{\mathcal{R}}}}
$$

The latter prescription II can have a chaotic inflation for $\xi \sim 10^{2}$, since the effectual potential (A15) becomes

$$
\mathcal{U} \sim \text { const }+\frac{\beta_{\lambda} M_{\mathrm{P}}^{2}}{48 \xi^{2}} \varphi^{2}
$$

due to

$$
\mathcal{G} \rightarrow \frac{\sqrt{6} M_{\mathrm{P}}}{\varphi}
$$

for large $\xi_{\mathcal{R}}$; see Appendix A.

(ii) When we have large $\xi_{\Phi}$ only, in particular with $F_{\mathcal{R}}=1$ which gives $U=V=\frac{\lambda}{4} \varphi^{4}$, we get

$$
\mathcal{G} \rightarrow \sqrt{\xi_{\Phi}} \frac{\varphi}{M}
$$

and hence

$$
\mathcal{U}=\text { const }+\frac{\lambda M^{2}}{2 \xi_{\Phi}} \varphi^{2} .
$$

This can also lead to a chaotic inflation when $\lambda / \xi_{\Phi} \ll 1[31]$.

(iii) When we terminate the tree-level potential at the $2 n$th order

$$
V=\sum_{n^{\prime}: \text { even, } 4 \leq n^{\prime} \leq 2 n} \lambda_{n^{\prime}} \frac{\varphi^{n^{\prime}}}{M^{n^{\prime}-4}},
$$

instead of Eq. (53), and the function at the $n$th order

$$
F_{\mathcal{R}}=\sum_{n^{\prime}: \text { even, } 0 \leq n^{\prime} \leq n} \xi_{\mathcal{R}, n^{\prime}} \frac{\varphi^{n^{\prime}}}{M^{n^{\prime}}},
$$

the resultant classical potential becomes constant [32]:

$$
U \rightarrow \frac{\lambda_{2 n}}{\xi_{\mathcal{R}, n}^{2}} M^{4}
$$

[1] D. S. Salopek, J. R. Bond, and J. M. Bardeen, Designing density fluctuation spectra in inflation, Phys. Rev. D 40, 1753 (1989).

[2] F. L. Bezrukov and M. Shaposhnikov, The standard model Higgs boson as the inflaton, Phys. Lett. B 659, 703 (2008).

[3] P. A. R. Ade et al. (Planck Collabortion), Planck 2015 results. XX. Constraints on inflation, Astron. Astrophys. 594, A20 (2016).

[4] J. J. van der Bij, Can gravity make the Higgs particle decouple?, Acta Phys. Pol. B 25, 827 (1994).

[5] J. L. Cervantes-Cota and H. Dehnen, Induced gravity inflation in the standard model of particle physics, Nucl. Phys. B442, 391 (1995).

[6] J. J. van der Bij, Can gravity play a role at the electroweak scale?, Int. J. Phys 1, 63 (1995).

[7] F. Lucchin, S. Matarrese, and M. D. Pollock, Inflation with a nonminimally coupled scalar field, Phys. Lett. 167B, 163 (1986).
[8] T. Futamase and K.-i. Maeda, Chaotic inflationary scenario in models having nonminimal coupling with curvature, Phys. Rev. D 39, 399 (1989).

[9] Y. Ema, R. Jinno, K. Mukaida, and K. Nakayama, Violent preheating in inflation with nonminimal coupling, J. Cosmol. Astropart. Phys. 02 (2017) 045.

[10] G. Aad et al. (ATLAS, CMS Collabortions), Combined Measurement of the Higgs Boson Mass in $p p$ Collisions at $\sqrt{s}=7$ and $8 \mathrm{TeV}$ with the ATLAS and CMS Experiments, Phys. Rev. Lett. 114, 191803 (2015).

[11] Y. Hamada, H. Kawai, and K.-y. Oda, Bare Higgs mass at Planck scale, Phys. Rev. D 87, 053009 (2013); Erratum, Phys. Rev. D89, 059901 (2014).

[12] Y. Hamada, H. Kawai, K.-y. Oda, and S. C. Park, Higgs inflation from standard model criticality, Phys. Rev. D 91, 053008 (2015).

[13] F. L. Bezrukov, A. Magnin, and M. Shaposhnikov, Standard Model Higgs boson mass from inflation, Phys. Lett. B 675 , 88 (2009). 
[14] F. Bezrukov and M. Shaposhnikov, Standard model Higgs boson mass from inflation: Two loop analysis, J. High Energy Phys. 07 (2009) 089.

[15] K. Allison, Higgs xi-inflation for the 125-126 GeV Higgs: A two-loop analysis, J. High Energy Phys. 02 (2014) 040.

[16] Y. Hamada, H. Kawai, K.-y. Oda, and S. C. Park, Higgs Inflation Still Alive, Phys. Rev. Lett. 112, 241301 (2014).

[17] F. Bezrukov and M. Shaposhnikov, Higgs inflation at the critical point, Phys. Lett. B 734, 249 (2014).

[18] B. J. Broy, D. Coone, and D. Roest, Plateau inflation from random non-minimal coupling, J. Cosmol. Astropart. Phys. 06 (2016) 036.

[19] Y. Hamada, H. Kawai, and K.-y. Oda, Minimal Higgs inflation, Prog. Theor. Exp. Phys. 2014, 23 B02 (2014).

[20] Y. Hamada, H. Kawai, and K.-y. Oda, Eternal Higgs inflation and the cosmological constant problem, Phys. Rev. D 92, 045009 (2015).

[21] A. Pomarol, Grand Unified Theories without the Desert, Phys. Rev. Lett. 85, 4004 (2000).

[22] D. P. George, S. Mooij, and M. Postma, Quantum corrections in Higgs inflation: The real scalar case, J. Cosmol. Astropart. Phys. 02 (2014) 024.

[23] D. P. George, S. Mooij, and M. Postma, Quantum corrections in Higgs inflation: The standard model case, J. Cosmol. Astropart. Phys. 04 (2016) 006.
[24] K. Kannike, G. Hutsi, L. Pizza, A. Racioppi, M. Raidal, A. Salvio, and A. Strumia, Dynamically induced Planck scale and inflation, J. High Energy Phys. 05 (2015) 065.

[25] M. Postma and M. Volponi, Equivalence of the Einstein and Jordan frames, Phys. Rev. D 90, 103516 (2014).

[26] A. Yu. Kamenshchik and C.F. Steinwachs, Question of quantum equivalence between Jordan frame and Einstein frame, Phys. Rev. D 91, 084033 (2015).

[27] N. Makino and M. Sasaki, The density perturbation in the chaotic inflation with nonminimal coupling, Prog. Theor. Phys. 86, 103 (1991).

[28] G. Domènech and M. Sasaki, Conformal frame dependence of inflation, J. Cosmol. Astropart. Phys. 04 (2015) 022.

[29] G. Domènech and M. Sasaki, Conformal Frames in Cosmology, Int. J. Mod. Phys. D 25, 1645006 (2016).

[30] J. Gomis and S. Weinberg, Are nonrenormalizable gauge theories renormalizable?, Nucl. Phys. B469, 473 (1996).

[31] K. Nakayama and F. Takahashi, Higgs chaotic inflation and the primordial B-mode polarization discovered by BICEP2, Phys. Lett. B 734, 96 (2014).

[32] S. C. Park and S. Yamaguchi, Inflation by non-minimal coupling, J. Cosmol. Astropart. Phys. 08 (2008) 009. 